\title{
Mobile Cloud Computing and Its Effectiveness in Business Organizations
}

\author{
Mohammad Awni Ahmad Mahmoud* \\ MIS Department \\ College of Applied Studies and Community Service, Imam Abdulrahman Bin Faisal University \\ KSA (2019) \\ Mohammed Abu Elaila Ali Baraka \\ MIS Department \\ College of Applied Studies and Community Service, Imam Abdulrahman Bin Faisal University
} KSA (2019)

Mohammed Fahed Ali Tayfour

MIS Department

College of Applied Studies and Community Service, Imam Abdulrahman Bin Faisal University

KSA (2019)

Abeer Atallah Mohammad Aloudat

Accounting Department

College of Applied Studies and Community Service, Imam Abdulrahman Bin Faisal University

KSA (2019)

\begin{abstract}
E-commerce business organizations aim at achieving the goals and the mission effectively and efficiently so as to satisfy the diverse interests of the stakeholders. MCC is an ICT concept that enables the organizations to enhance the performance when serving the important stakeholders, who include customers, staff, managers, shareholders, and industry regulators. MCC involves the integration of the mobile devices to enable the sharing of the cloud infrastructure. The integration is done via a network and between the computer devices that operate remotely. The Internet is the most common network that enables the mobile devices to utilize the data and information stored in a cloud database. E-commerce businesses prefer the cloud infrastructure because it has a large data storage capacity and high processing speeds. Also, the cloud service providers invest substantial financial, human, and technological resources in ensuring the security of the effective management of the data resources. The main benefit of MCC is that it reduces the businesses expenses. For example, it enables the companies to offer products and services in the international market via the e-commerce infrastructure. Amazon.com is an example of a Multinational Corporation that is successful in offering high-quality services and products to customers in different countries using the website and the mobile app applications that are supported by the cloud infrastructure.
\end{abstract}

Keywords: mobile cloud computing, cloud computing, E-commerce.

DOI: $10.7176 / \mathrm{IKM} / 9-1-04$

\section{Introduction}

Mobile Cloud Computing (MCC) is an important innovation in the Information and Communication Technology (ICT) sector of the economy. It implies the integration of the critical technologies to generate maximum effectiveness or output in the ICT systems. The necessary technologies comprise cloud computing, wireless networks, and mobile computing. These technologies operate together by sharing resources and capabilities to improve the utility of the system users, providers of cloud computing services, and computer network providers (Nagpal et al., 2017). The key objective of MCC is to develop and enhance the utilization of the innovative mobile applications on diverse mobile devices. MCC offers significant economic and performance benefits to the individual and organizational users, and also the service providers like the mobile network and the cloud services providers. MCC illustrates the mobile computing systems that utilize various cloud and network resources to enhance the functionality, mobility, and memory of the mobile devices via the Internet or the Ethernet. It operates effectively despite the heterogeneous and the network environment and the pay-as-you-use principle.

MCC applies the concept of computational augmentation process as the utilization is done on remote devices, as opposed to one system. Thus, the mobile resources can care the computation resources like various cloud and network services remotely. There are three major cloud resources that are applicable to the area of mobile computing. These are distant immobile, proximate immobile, and proximate mobile entities. The most effective MCCC platform utilizes an integration or hybrid of the three computing platforms, to enhance the 
sharing of resources, capabilities, and networks remotely. There are various Multinational Corporations that offer MCC services to individuals and organizations. Vodaphone and Orange are the leaders in the MCC sector and they partner with different organization globally in order to enhance technological innovation and business performance. An example of a giant cloud is the Amazon EC2 and it operates using the immobile computing model (Desai, 2016). Also, the cloudlet platform operates using the proximate immobile platform. Additionally, the handheld devices like tablets and smartphones utilize the proximate mobile platform.

MCC offers functions and capabilities that enhance the development and the utilization of the next generation applications, especially in the competitive and innovative business environment. MCC has a significant influence on the traditional e-commerce, as it offers effective solutions for the improvement of the online business systems, processes, and transactions. MCC highly depends on the mobile applications as the accessibility of the Internet-enabled handheld devices is increasingly at a high rate globally. Through the incorporation of MCC via the mobile devices and the Internet, the business organizations get the advantages of low business expenses, wide market reach, high sales volumes and revenues, and proper management of customer data (Raja, Chitra \& Jonafark, 2018). Therefore, MCC is an important concept in enhancing the ecommerce systems and platforms of the business organizations.

\section{Problem Statement}

The mobile application is important in the current society. It enables e-commerce business organizations to engage in various activities that improve the delivery of products and services to the customers. For example, mobile application enables the users to engage in online shopping activities on various e-commerce platforms; for example, Amazon.com and Alibaba.com. But, the mobile application has several challenges. It has low data bandwidth, ineffective security features, limited processing capabilities, and low storage levels. It is easy to steal and use the data of the customers through the mobile application. Thus, there is the need for the business and the ICT stakeholders to develop solutions so as to enhance the performance and satisfaction of the stakeholders of the organization. MCC is an effective approach to overcoming all the shortcomings of the mobile applications. It enables the business to benefit from a large storage space that is secure. The cloud storage service ensures data confidentiality and authorized access. For example, the e-commerce business can store the transaction history of all the customers through the cloud computing infrastructure. MCC ensures on-demand access to the data and the information in the cloud application. The authorized individuals can access any information at any time, as the cloud service providers are in the business of storing and retrieving the data of the clients effectively and efficiently.

Cloud computing has fast computation abilities. The data management processes are executed immediately or in real-time. After accessing the cloud system, the client will have the on-demand access to information. MCC utilizes innovative security features. For example, there is the two-factor authentication feature that enables a security feature to be accessible on several devices before granting access to the data system. Mobile computing ensures remote access to the network or database of an organization (Hill, 2017). For example, the client can access his or her account in the customer portal of the e-commerce website from any location. Amazon.com has customers from different countries that access the shopping services through the client accounts in the customer portal.

E-business offers the infrastructure that enables the clients to engage in different transactions online. The integration of MCC and business intelligence is important in generating new opportunities and improving the effectiveness of the organization. MCC enables the business organizations to manage huge data in a secure, mobile, and accessible manner. SaaS is an example of a mobile application. Its effectiveness is enhanced by the cloud infrastructure and, therefore, it can be utilized anywhere and from different handheld devices. SaaS organizations; for instance, Salesforce.com operate by offering mobile platforms for the diverse services. There is a significant migration from the mobile platforms from the devices towards the back-end servers. Apple and Google are examples of the corporations that offer the computing and the storage services on their cloud infrastructure. Hence, MCC offers benefits like minimal ICT costs and offers the network infrastructure that enhances the operations of the business. Mobile commerce is an increasing phenomenon in the business environment as more organizations interact with their customers through the various online and internet platforms. Small and Medium Enterprises (SME) benefit from the law services offered by the MCC service providers; for example, they can store their data securely on the cloud platforms (Shanklin, 2018). Also, the large organizations benefit from the sufficient ICT resources offered by the MCC infrastructure. Customers and staff in different locations can communicate effectively using the mobile and cloud-based systems.

\section{Research Questions}

a. What is the meaning of MCC in the context of the e-commerce company environment?

b. What are the applications of MCC in the activities of the e-commerce business organization?

c. How can MCC enhance the performance or effectiveness of the e-commerce company? 
d. What are the emerging practices and innovations in the area of MCC in the e-commerce sector?

e. What is the SWOT analysis of MCC in the context of the e-commerce business environment?

\section{Research Objectives}

a. To understand the concept of MCC as applicable in the e-commerce sector of the economy.

b. To illustrate the uses of MCC by the company that engages in the online sale of high-quality products and services.

c. To show how MCC improves the productivity of the company that engages in the online business transactions.

d. To shows the new ideas and concepts in the area of MCC and the applications in the online business concepts and transactions.

e. To conduct a SWOT analysis of MCC with the intention of understanding the internal and external business environment of the e-commerce company.

\section{Scope of the Study}

MCC is a development of the cloud and mobile computing infrastructures and systems. Mobile computing allows the transmission of data through the wireless devices. The devices include computers, smartphones, smartwatches, and tablets. In mobile computing, there is no physical connection of the devices. Companies use the mobile computing infrastructure. For example, the staff share work reports using their smartphones through the email and the social media platforms. Most corporations are adopting applications that can support several mobile operating systems. For example, the devices can have online and Bluetooth connectivity that offers remote computing services (Natkar, 2016). There are two requirements for the effective operation of the mobile computing. The first is processing power, which should be sufficient to achieve the proper processing and transfer of data. The second is the memory, which should be sufficient to achieve sufficient storage of the data. However, the two key issues affecting the mobile applications are data security and insufficient storage. Therefore, there is the need to enhance the mobile application by combining it with the effective cloud computing platform.

Cloud computing comprise the remote servers that can access centralized data storage services, online infrastructure, and common ICT infrastructure. There are three categories of the cloud: private, public, and hybrid. The computer application operates by having all the processes and storing all the data online to ensure effective on-demand access via the mobile devices. The applications operate on remote servers. All the processing is done by the remote applications and the result are effectively transmitted to the user devices. There is significant flexibility in the cloud computing services as the users access appropriate information and utilize it according to his or her unique needs and interests. For example, the user can utilize the cloud computing services at all times by granting 24/7 access (Arvind \& Manimegalai, 2017). Alternatively, he or she can utilize the cloud services periodically by providing limited access that can be in seconds, minutes, or hours.

MCC enables the mobile device to conduct the data storage and processing activities. It transfers the data and processing functions from the mobile device to the centralized computing system that are located in the clouds through the wireless connection. There are several challenges that affect the mobile devices; for instance, low battery life, insufficient bandwidth, and limited storage space. Thus, MCC provides the user with sufficient infrastructure at an affordable cost and with flexible usage platforms. It has a large storage capacity and high processing abilities (Meng, 2017). The connection of the device to the cloud system is simple as it is done through the wireless connection like the Internet. It enables the businesses to expand their operations globally as the customers and the staff can access important services and facilities through the devices that are connected to the cloud system.

The study investigates how an e-commerce company can use the MCC infrastructure effectively and efficiently. It also demonstrates the key problems that hinder the e-commerce organization from integrating all its activities in the cloud infrastructure. The e-commerce company or corporation is a business organization that relies on the computer network or the Internet in the provision of services and products to the customers in different countries. The dominant e-commerce companies in the present business environment include Amazon.com, Alibaba.com, eBay.com, and Walmart.com. Customers create an account on the website or mobile applications of the e-commerce company. The personal account facilitates the activities of selling or purchasing products online. The orders are placed, payments made, and then the product is shipped to the addresses of the customers affordably, conveniently, and timely. MCC infrastructure improves the e-commerce activities as it ensures the storage of large volume of information securely and confidentially (Natkar, 2016). MCC also enhances the processing speed and, therefore, the e-commerce staff and customers access and retrieve data in real-time.

The problems that affect the complete adoption of MCC by the e-commerce company are diverse. First, the mobile devices of the staff and customers like the smartphones and tablets have limited energy source. The 
customers cannot access the website or the mobile app if the batteries of the devices have insufficient energy levels. Therefore, it is essential for the customers to perform online transactions when the batteries have full energy capacity. Second, most of the e-commerce activities are done using wireless connectivity as the devices are portable or handheld. Wireless connectivity experiences intermittent connectivity and variable data levels because of the gaps in network coverage, more so in remote regions. Uncontrollable issues like extreme weather changes can cause network disconnection, which hinders the customers from performing online transactions. Thus, the e-commerce company should focus its activities in areas that have both fixed broadband and wireless bandwidth to ensure maximum internet connectivity. Third, the mobile devices have less resource capacity than the fixed devices like the Personal Computers. For example, mobile devices have low memory capacity and processing speeds (Abadir, Schock, \& Maffett, 2017). Also, the network bandwidth is insufficient resulting in a poor network connection. To address the challenges, the cloud service providers should invest in online resources that enhance the effectiveness of the mobile devices. Therefore, it is important for the e-commerce company to understand the problems of MCC adoption and develop practical solutions with the assistance of the cloud service providers.

\section{Significance of the Study}

There are popular cloud services that the business organizations can utilize to expand the communication and data management processes. An example of the cloud service is the Amazon Web Service. It offers the Infrastructure as a Service (IaaS) platform that enables business organizations to store large data. It also provides the Platform as a Service (PaaS) that enables the organizations to utilize the cloud infrastructure of Amazon at an affordable price. Many ICT developers are exploring more ways to improve the customization of the cloud services and infrastructure to enhance the utility and satisfaction of the users. For example, the customization enables an automobile company to store unique content that enhances the quality and innovation in the motor vehicle sector of the economy. Likewise, a hotel will require customization to manage the hospitality information and the customer data effectively and efficiently.

MCC improves the e-business processes. It offers a platform for the effective storage and processing of data and information that enhance the customer transactions. E-business takes place over a secure electronic network that facilitates effective information processing and exchange. There are various classifications of the e-business transactions through the MCC platforms. There is the business-to-business transaction that enhances the exchange of information between corporates; for example, between the payment service provider like PayPal or MasterCard and the e-commerce site like eBay.com or Alibaba.com. Business-to-consumer transactions entail communication from the organization to the clients. For example, the email messages from the company that informs the customers about the availability of superior products and services. Consumer-to-consumer transactions facilitate information exchange between the organizational customers. For example, the customers can exchange the product review information through the e-commerce network. Consumer-to-business illustrates the information and the data that the clients enter in the network of the company. For example, the customer can enter payment information to facilitate the online purchase process. Also, the customer can place new orders to show the desire to purchase a product or service.

The e-business system covers all business processes or activities, from the development of the product to the purchase and review of the product by the customers. There is product development software that enables the business to design high-quality products. For example, in the automobile sector, computer software enables the designers to develop energy efficient cars and even the self-driving vehicles that are environmentally sound. In product marketing, the e-commerce system can communicate useful information about the products or the services to the customers. For example, the website and the social media platforms can communicate the value of a new product in the market. Product selling is done online as the customer places an order electronically via the website or the mobile app. There are various online payment options; for example, MasterCard, VISA card, Payoneer, PayPal, and cryptocurrencies like bitcoin. There are many categories of e-business. For example, eworking enables the staff to work from home or from remote locations. A sales staff can submit the daily revenue records through the website of the company (Abadir, Schock, \& Maffett, 2017). Qualified individuals are also allowed to apply for vacancies in the organization through the e-recruitment portal. E-marketing enables the business to use the effect of the Internet, social media, and emails to inform current and potential customers about the availability of a product or service.

\section{Research Limitations}

The research topic is complex. It can be understood by the individuals who study or practice in the ICT field. However, the ordinary individuals can have challenges in understanding the computer terminologies. For example, MCC enables the computer and mobile devices to be connected remotely with the objective of sharing the cloud infrastructure. The connection is done online. As a result, there is the Internet of Things (IoT) and the Internet of Everything ( $\mathrm{IoE}$ ) concepts that signify the increasing role of online connectivity in the society 
(Tawalbeh et al., 2017). Many people access crucial services online. For example, they purchase products and services through the e-commerce platforms like eBay.com. MCC infrastructure operates through Software as a Service (SaaS) platform. The numerous transactions that they engage in are offered by the cloud service providers. Therefore, ordinary people cannot understand the complex terminologies that are used in the description of the MCC systems in the research.

The research duration is limited. There is the need for more time, especially in the data collection process. The available time is only sufficient for the data mining process of data collection, as there are scholarly books and articles on the MCC topic in the various ICT online databases. However, it is important to collect information from the professionals in the ICT and business intelligence fields of practice and study. But, collecting information from the respondents through interviews and questionnaires requires sufficient time.

The research area is too broad, as there are many applications of MCC in the business environment. In fact, the high rates of the adoption of the IoE and IoT in the society increase the number of activities that are performed using the MCC platform. All sectors of the economy use the internet to perform various activities remotely. A farmer uses the smartphone to research the price of various vegetable products in a market. An Uber driver uses the google map application to identify the location and the destination of the customers. A musician uses the YouTube app to monitor the popularity of his or her music video. The applications of MCC are diverse in all the sectors of the economy, and hence they present a broad area of research.

\section{Literature Review}

Business activities are more effective when they are performed using the MCC platform. The $3 \mathrm{G}$ and the $4 \mathrm{G}$ Mobile service providers ensure that there is effective Internet connection to facilitate the remote business transactions. E-commerce ensures on-demand services and, therefore, the needs and interests of the customers are achieved immediately. For example, a customer can identify the available products and services after accessing the customer portal on the business website. Many business activities are facilitated by the MCC infrastructure. For example, the electronic money transfer services operate through the secure and confidential cloud infrastructure. Also, the online ticketing process is facilitated by the MCC systems (Abadir, Schock \& Maffett, 2017). There are algorithms that facilitate the effectiveness of the business processes. For example, the recommendation algorithm utilizes the mathematical analysis of the previous customer transactions to monitor the loyalty levels.

There are several advantages of the MCC processes in the businesses. It improves cost efficiency, and as a result, the businesses record more revenues. There is cost effective as the businesses are not required to invest in the software and hardware. All there data processing needs are handled by the cloud service providers. They pay minimal fees in order to access the cloud services on a regular basis. The organization can start or expand the online businesses with minimal investment risks as all the data analysis on the market will be done by the cloud system. For example, the cloud system can generate important information on the economies that have a high demand for the products or services of the organization. MCC gives the users the access to more information on their mobile devices. For example, through the websites and the mobile apps, the users can perform various online activities that include shopping, research, and entertainment. MCC gives the business sufficient access to the large markets. Amazon.com has a global customer base with minimal investments in physical infrastructure as the customers can perform all shopping transactions through the website and the mobile app platforms. MCC has fast transactions due to the effective $3 \mathrm{G}$ and $4 \mathrm{G}$ online services (Oshima et al., 2018). Quick internet connectivity enables the users to conduct several business transactions or simultaneous activities. For example, a user can request for an e-book from Alibaba.com, while listening to YouTube music from the same device.

However, there are business issues that are associated with the MCC platforms. The online business transactions cannot occur when there is an ineffective or unavailable network connection. The mobile cloud application can only be accessed online. Unstable Internet connection limits the access and use of the cloud services. Power failures and atmospheric disturbance can limit the effectiveness of the online network. The users who are in the high-rage sites from the cloud services infrastructure experience poor performance. The highlatency in the online connection causes delays in the information processing. There are data security concerns in the use of the cloud systems. Many users fear that their data may be accessed or destroyed by the unauthorized individuals or viruses, especially if the network does not have effective firewall protection. The cloud service providers have full control of the data management business, as the individual and corporate users do not benefit from customized or specific systems (Turban et al., 2018). The cloud service providers develop a general system that is used by organizations from different sectors of the economy. There are high bandwidth costs when using the cloud services. Large data processes attract high network bandwidth fees.

There are several mobile commerce services that utilize the cloud computing applications. Billing services occur through the cloud computing platform. The mobile payments are authentic and they are done in a secure system that protects the user data. Thus, businesses and users perform various payment transactions through the cloud platform. Online shopping is an e-commerce activity that occurs effectively through the cloud platform. 
The customers identify and pay for products and services online. Real-time tracking of the shipping process enhances the safety of the purchased products. The tracking is done using the information exchange between the product sensors and the satellite communication systems that are managed by the cloud service providers. Business alerts are managed through the MCC platform. For example, shareholders can receive daily price alerts on the price movements. MCC manages the advertisement activities. For example, the business can communicate big sale information through the MCC platform by sending text, email, and social media messages. The auction is a business process that is facilitated by MCC. People from different countries can purchase a product in a warehouse in the New York City through the automated auction system (Balaji, Nagaraju, \& Haritha, 2017). The virtual auction is also critical in the online purchase and sale of the cryptocurrencies. Online forex is an activity that is controlled by the MCC. Users in different countries purchase and sell currencies through the website of the Forex company in real-time.

\section{Methodology}

The research methodology is mixed. It involves using the quantitative and the qualitative techniques in the collection, analysis, and presentation of data. The mixed research methodology is effective in offering explanations and supportive data on the applications of MCC in the improvement of various business processes. The data mining process will generate both qualitative and qualitative information. Qualitative information expresses a thought. For example, MCC enables companies to manage the big data effectively and efficiently in order to achieve a competitive advantage in the competitive business environment. Quantitative data offers statistical evidence. For instance, in 2014, approximately 1.3 billion smartphones were purchased in the United States (Benitez et al., 2018). Thus, the high levels of ownership of the mobile devices enhance the applications of CMM in the organizational environment. Employees and the managers communicate effectively through the online platforms of email, social media, and websites.

Data analysis is done using both qualitative and quantitative approaches. Qualitative analysis shows theoretical support. For instance, business organizations should embrace MCC as it minimizes the cost of operations. The support is positive as the adoption of e-commerce is not capital intensive when compared to the development of physical infrastructure in different countries. Quantitative data analysis is done using the statistical measures of central tendencies and dispersion. For instance, the mode is a measure of central tendency that determines the industry or economic sector that has the highest number of companies that use the MCC platform to drive innovation and productivity (Jones et al., 2017). In the discussion, it is the ICT industry that has the highest number of corporations that implement the MCC services. The technology giants like Facebook, Amazon.com, and Uber use the MCC strategy in the management of the data of the stakeholders. Uber allows the customers to request for travel services via the Uber mobile app. The current location of the customer and the destination site are calculated automatically using the Google map platform (Ahern, 2017). The range is the measures of dispersion that will be applicable in determining the differences in the performance of companies before and after the adoption of the MCC platform. The research assumption is that the MCC systems enhance the performance of the organization as it improves the market dominance and reduces the cost of doing business.

The presentation of data will be done using qualitative and quantitative methods. The qualitative results will be recorded in tables and discussions. However, the quantitative results or findings will be presented in charts and graphs. For instance, the performance trends of the companies using the MCC platforms will be illustrated in charts and graphs to illustrate the relationships between the dependent and the independent variables. Charts and graphs are important quantitative instruments that compare the performance values of organizations. The image below illustrates a bar graph that illustrates the growing significance of cloud application in the mobile data traffic (Natkar, 2016). 


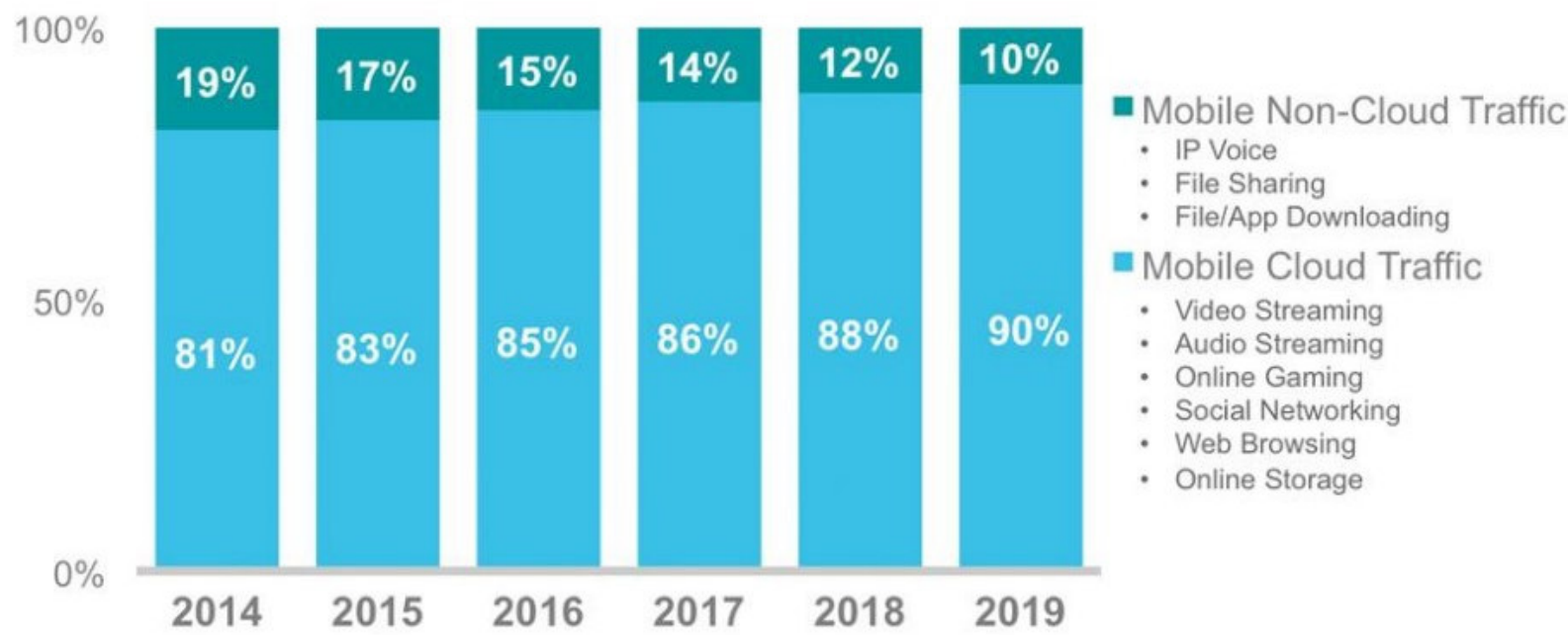

Source: Cisco VNI Mobile, 2015

\section{Research Framework}

The research framework is based on SWOT analysis. It is important to understand the strengths, weaknesses, opportunities, and threats that the MCC platform has on the internal and the external environment of the various business organizations. The strengths illustrate the internal capabilities that a company gains by using MCC. The company will be effective in the management of the data resources. As a result, the staff will understand the needs and the interests of the customers. The management will be able to identify the product or service that has the highest demand levels. Thus, they will supply the highest quality of the product or service in order to maximize profits and customer satisfaction. For example, Uber concentrates most of its traveling services in the urban areas of the developed economies as the demand for the service is high. Also, MCC enables the company to increase its revenues by reducing the cost of doing business. Amazon.com has low business expenses because most of its business activities are conducted globally through the website (Parvinen et al., 2017). As a result, its performance is better than the traditional brick-and-mortar shopping outlets like Walmart and Carrefour.

The weaknesses illustrate the challenges that MCC causes in the internal business environment. For example, there are employees who are resistant to change. They view the adoption of new technology in the business environment as a threat to their employment security. For example, the sales staff may resist the adoption of the e-commerce system as the online sales may render their service in the organization obsolete. The internet or bandwidth charges of using the cloud infrastructure are high due to the high volume of data (Guo et al., 2017). Also, the initial cost of installing the MCC applications is high and thus may reduce the revenues of the company at the initial stages of development and implementation.

MCC presents tremendous opportunities for the development and expansion of the business activities. Companies that adopt e-business or e-commerce have the change of expanding their operations overseas. For instance, eBay.com is an American corporation that began operations in the United States. But, the e-commerce platform has enabled it to get loyal customers in other countries. Also, MCC improves innovation in the business environment. Google is currently developing the self-driving Google Cars that will operate using the data in the cloud systems (Andre et al., 2018). The automated care will be electric and it will have sensors that prevent collision with objects and hence improving road safety and safeguarding the natural environment.

There are threats to the adoption of MCC in the business sector. The first threat is data security. Many businesses have concerns that unauthorized individuals or malicious programs may access or destroy the data that they store in the cloud system. Thus, these companies spend significant financial resources in developing a physical backup system for all electronic data. The human resource budget is high as the company must higher competent data security experts and systems administrators to ensure that all electronic data are safe (Benedetti et al., 2017). Also, there are legal challenges associated with the adoption of the MCC infrastructure. For instance, Uber has been banned from operating in several countries like Germany and China due to the insufficient legal framework to guide its operations and generate revenues for the government. 


\begin{tabular}{|l|l|}
\hline Strengths & Weaknesses \\
\hline Effective management of data resources & Threat to employment security \\
Increase business revenues & High cost of bandwidth or Internet services \\
\hline Opportunities & Threats \\
\hline Business expansion through e-commerce & Data security \\
Enhances effective innovation & Legal challenges \\
\hline
\end{tabular}

\section{Conclusion and Expected Output}

MCC is an important development in the business environment as it aims at improving the performance. It offers effectiveness in the management of the large data resources of the organization as the data processing infrastructure is provided by the competent cloud service providers. MCC drives innovation as it enables the corporations to develop and implement new ideas that are critical in the development of high-quality products and services that achieve the needs and the expectations of the customers. There is the need to enhance the confidence of the MCC users in the area of data security. The cloud service providers must adopt effective data and system security measures that prevent the malicious access and usage of the data and information of the individual and corporate clients.

\section{References}

Desai, N. S. (2016). Mobile cloud computing in business. International Journal of Information, 6(1/2). Retrieved from http://aircconline.com/ijist/V6N2/6216ijist21.pdf

Raja, C. V., Chitra, K., \& Jonafark, M. (2018). A Survey on Mobile Cloud Computing. International Journal of Scientific Research in Computer Science, Engineering and Information Technology, 3(3). Retrieved from http://ijsrcseit.com/paper/CSEIT18354.pdf

Hill, D. C. (2017). U.S. Patent No. 9,658,868. Washington, DC: U.S. Patent and Trademark Office. Retrieved from https://patents.google.com/patent/US9658868B2/en

Shanklin, M. (2018). Mobile Cloud Computing. Retrieved from https://www.cse.wustl.edu/ jain/cse574$10 / \mathrm{ftp} /$ cloud/index.html

Natkar, S. (2016). Mobile Cloud Computing: The Upcoming Trend! Retrieved from https://www.esds.co.in/blog/mobile-cloud-computing-the-upcoming-trend/\#sthash.JoewUC83.dpbs

Arvind, K. S., \& Manimegalai, R. (2017). Secure data classification using superior naive classifier in agent based mobile cloud computing. Cluster Computing, 20(2), 1535-1542. Retrieved from https://www.igiglobal.com/article/secure-mobile-multi-cloud-architecture-for-authentication-and-data-storage/179538

Meng, T. (2017). Security and Performance Tradeoff Analysis of Offloading Policies in Mobile Cloud Computing (Doctoral dissertation, Freie Universität Berlin). Retrieved from https://dnb.info/1136903623/34

Tawalbeh, L. A. A., Ababneh, F., Jararweh, Y., \& AlDosari, F. (2017). Trust delegation-based secure mobile cloud computing framework. International Journal of Information and Computer Security, 9(1-2), 36-48. Retrieved from https://www.inderscienceonline.com/doi/pdf/10.1504/IJICS.2017.082838

Abadir, E. E., Schock, R., \& Maffett, I. D. (2017). U.S. Patent No. 9,779,393. Washington, DC: U.S. Patent and Trademark Office. Retrieved from https://patents.google.com/patent/US9779393B2/en

Oshima, M., Hayashi, K., Kageyama, S., Sakashita, S., Isogawa, T., \& Takayama, H. (2018). U.S. Patent Application No. 14/559,399. Retrieved from https://patents.google.com/patent/USRE46775E1/en

Turban, E., Outland, J., King, D., Lee, J. K., Liang, T. P., \& Turban, D. C. (2017). Electronic commerce 2018: a managerial and social networks perspective. Springer. Retrieved from https://books.google.co.ke/books?hl=en\&lr=\&id=D9Q5DwAAQBAJ\&oi=fnd\&pg=PR5\&dq=ecommerce+applications\&ots=InNRHvoHVS\&sig=PzNUnq35RVSFgyI4Z7vjgnP0x8I\&redir_esc $=y \# v=$ one page \&q=e-commerce $\% 20$ applications $\& \mathrm{f}=$ false

Balaji, P., Nagaraju, O., \& Haritha, D. (2017). Reputation Based Trust Evaluation in E-Commerce Applications by Using Feedback Comments. Retrieved from http://www.ijcseonline.org/pub_paper/8-IJCSE-01934.pdf

Benitez, J., Chen, Y., Teo, T. S., \& Ajamieh, A. (2018). Evolution of the impact of e-business technology on operational competence and firm profitability: A panel data investigation. Information \& Management, 55(1), 120-130. Retrieved from https://www.sciencedirect.com/science/article/pii/S0378720617306857

Ahern, M. (2017). U.S. Patent No. 9,619,766. Washington, DC: U.S. Patent and Trademark Office. Retrieved from https://patents.google.com/patent/US9619766B2/en

Parvinen, P., Kaptein, M., Pöyry, E., \& Hamari, J. (2017, January). Introduction to Customer Analytics and Data-Led Omnichannel Commerce Minitrack. In Proceedings of the 50th Hawaii International Conference on System Sciences. from https://scholarspace.manoa.hawaii.edu/bitstream/10125/41624/1/paper0475.pdf

Guo, L., Wei, Y. S., Sharma, R., \& Rong, K. (2017). Investigating e-business models' value retention for start- 
ups: The moderating role of venture capital investment intensity. International Journal of Production Economics, 186 $33-45$. Retrieved

from https://www.sciencedirect.com/science/article/pii/S0925527317300300

Andre, H. G., Carson, M. D., Chandra, R., Hardy, C. A., Juarez, L., Leung, T., \& Sorenson, T. C. (2018). U.S. Patent No. 9,946,618. Washington, DC: U.S. Patent and Trademark Office. Retrieved from https://patents.google.com/patent/US9946618B2/en

Benedetti, F., Di Cocco, A., Marinelli, C., \& Pichetti, L. (2017). U.S. Patent No. 9,591,074. Washington, DC: U.S. Patent and Trademark Office. Retrieved from https://patents.google.com/patent/US9591074B2/en

Jones, S., Irani, Z., Sivarajah, U., \& Love, P. E. (2017). Risks and rewards of cloud computing in the UK public sector: A reflection on three Organisational case studies. Information Systems Frontiers, 1-24. Retrieved from https://link.springer.com/content/pdf/10.1007/s10796-017-9756-0.pdf

Nagpal, A. R., Patil, S. R., Ramanathan, S., Shukla, D., \& Trevathan, M. B. (2017). U.S. Patent No. 9,712,495. Washington, DC: U.S. Patent and Trademark Office. Retrieved from https://patents.google.com/patent/US9712495B2/en 